8. Halstead SB. Chikungunya. In: Feign RD, Cherry J, Demmler-Harrison GJ, Kaplan SL, eds. Feign and Cherry's Textbook of Pediatric Infectious Diseases. Philadelphia, PA: Saunders Elsevier; 2004:2178-2184.

9. Enserink M. Epidemiology. Tropical disease follows mosquitoes to Europe. Science. 2007;317(5844):1485.

10. Myers RM, Carey DE, Reuben R, Jesudass ES, De Ranitz C, Jadhav M. The 1964 epidemic of denguelike fever in South India: isolation of chikungunya virus from human sera and from mosquitoes. Indian J Med Res. 1965;53(8):694-701.

11. Anderson CR, Singh KR, Sarkar JK. Isolation of chikungunya virus from Aedes aegypti fed on naturally infected humans in Calcutta. Curr Sci. 1965;34:579-580.

12. Simon F, et al. Chikungunya infection; an emerging rheumatism among travelers returned from Indian Ocean Islands. Report of 47 cases. Medicine. 2007;86(3):123-137.

13. Schuffenecker I, et al. Genome microevolution of chikungunya viruses causing the Indian Ocean outbreak. PLoS Med. 2006;3(7):e263.

14. de Lamballerie X, Leroy E, Charrel RN, Tsetsarkin K, Higgs S, Gould EA. Chikungunya virus adapts to tiger mosquito via evolutionary convergence: a sign of things to come? Virol J. 2008;5:33.

15. Tsetsarkin KA, Vanlandingham DL, McGee CE, Higgs $S$. A single mutation in chikungunya virus affects vector specificity and epidemic potential. PLoS Pathog. 2007;3(12):e201.

16. Tsetsarkin KA, McGee CE, Volk SM, Vanlandingham DL, Weaver SC, Higgs S. Epistatic roles of E2 glycoprotein mutations in adaption of chikungunya virus to Aedes albopictus and Ae. aegypti mosquitoes. PLoS One. 2009;4(8):e6835.

17. Benedict MQ, Levine RS, Hawley WA, Lounibos LP. Spread of the tiger: global risk of invasion by the mosquito Aedes albopictus. Vector Borne Zoonotic Dis. 2007;7(1):76-85.

18. Gould EA, Higgs S. Impact of climate change and other factors on emerging arbovirus diseases. Trans R Soc Trop Med Hyg. 2009;103(2):109-121.

19. Lidbury BA, et al. Macrophage-derived proinflammatory factors contribute to the development of arthritis and myositis after infection with an arthrogenic alphavirus. J Infect Dis. 2008;197(11):1585-1593.

20. Ziegler SA, Lu L, da Rosa AP, Xiao SY, Tesh RB. An animal model for studying the pathogenesis of chikungunya virus infection. Am J Trop Med Hyg. 2008;79(1):133-139.

21. Wang $E$, et al. Chimeric alphavirus vaccine candidates for chikungunya. Vaccine. 2008;26(39):5030-5039.

22. Couderc T, et al. A mouse model for Chikungunya: young age and inefficient type-I interferon signaling are risk factors for severe disease. PLoS Pathog. 2008;4(2):e29.

23. Taubitz W, et al. Chikungunya fever in travelers: clinical presentation and course. Clin Infect Dis. 2007;45(1):e1-e4

24. Dubrulle M, Mousson L, Moutailler S, Vazeille M, Failloux AB. Chikungunya virus and Aedes mosquitoes: saliva is infectious as soon as two days after oral infection. PLoS One. 2009;4(6):e5895.

25. Boppana VD, Thangamani S, Adler AJ, Wikel SK. SAAG-4 is a novel mosquito salivary protein that programmes host CD4 T cells to express IL-4. Parasite Immunol. 2009;31(6):287-295.

26. Schneider BS, Soong L, Girard YA, Campbell G, Mason P, Higgs S. Potentiation of West Nile encephalitis by mosquito feeding. Viral Immunol. 2006;19(1):74-82.

27. Binn LN, Harrison VR, Randall R. Patterns of viremia and antibody observed in rhesus monkeys inoculated with chikungunya and other serologically related group A arboviruses. Am J Trop Med Hyg. 1967;16(6):782-785.

28. Paul SD, Singh KR. Experimental infection of Macaca radiata with Chikungunya virus and transmission of virus by mosquitoes. Indian J Med Res. 1968;56(6):802-811.

29. Chen C-I, et al. Development of a macaque model for in utero chikungunya virus infection. In: American Society of Tropical Medicine and Hygiene 58th Annual Meeting; November 18-22, 2009:Washington, DC. Abstract 2997.

30. Akahata W, et al. A virus-like particle vaccine for epidemic Chikungunya virus protects nonhuman primates against infection [published online ahead of print January 28, 2010]. Nat Med. doi: $10.1038 / \mathrm{nm} .2105$.

31. Rulli NE, et al. Ross River virus: molecular and cellular aspects of disease pathogenesis. Pharmacol Ther. 2005;107(3):329-342.

32. McIntosh BM, Peterson HE, McGillivray GM, de Sousa J. Further studies on the chikungunya outbreak in southern Rhodesia in 1962. I. Mosquitoes, wild primates and birds in relation to the epidemic. Ann Trop Med Parasitol. 1964;58:45-51.

33. McIntosh BM, Harwin RM, Paterson HE, Westwater ML. An epidemic of Chikungunya in South-Eastern Southern Rhodesia. Cent Afr J Med. 1963;43:351-359.

34. Inoue $\mathrm{S}$, et al. Distribution of three arbovirus antibodies among monkeys (Macaca fascicularis) in the Philippines. J Med Primatol. 2003;32(2):89-94.

35. Marchette NJ, Rudnick A, Garcia R, MacVean DW. Alphaviruses in Peninusular Malaysia: I. Virus isolations and animal serology. Southeast Asian J Trop Med Public Health. 1978;9(3):317-329.

36. Peiris JS, Dittus WP, Ratnayake CB. Seroepidemiology of dengue and other arboviruses in a natural population of toque macaques (Macaca sinica) at Polonnaruwa, Sri Lanka. J Med Primatol. 1993;22(4):240-245.

37. Cawthon Lang KA. Primate factsheets: Long-tailed macaque (Macaca fascicularis) taxonomy, morphology, \& ecology. http://pin.primate.wisc.edu/ factsheets/entry/long-tailed_macaque/taxon. Updated January 6, 2006. Accessed January 28, 2010.

38. Chastel C. Chikungunya virus: its recent spread to the southern Indian Ocean and Reunion Island (2005-2006) [in French]. Bull Acad Natl Med. 2005;189(8):1827-1835

\title{
Lights on for aminopeptidases in cystic kidney disease
}

\author{
Erwin P. Böttinger
}

Charles R. Bronfman Institute for Personalized Medicine, and Department of Medicine, Mount Sinai School of Medicine, New York, New York.

\begin{abstract}
While erudite cell biologists have for many decades described singular immotile appendages known as primary cilia to be present on most cells in our bodies, cilial function(s) long remained an enigma. Driven largely by an ever increasing number of discoveries of genetic defects in primary cilia during the past decade, cilia were catapulted from a long lasting existence in obscurity into the bright spotlight in cell biology and medicine. The study by O'Toole et al. in this issue of the JCI adds a novel "enzymatic" facet to the rapidly growing information about these little cellular tails, by demonstrating that defects in the XPNPEP3 gene, which encodes mitochondrial and cytosolic splice variants of $X$-prolyl aminopeptidase 3 , can cause nephronophthisis-like ciliopathy. Future studies are in order now to elucidate the cystogenic pathways affected by disrupted enzymatic function of XPNPEP3 in cilia-related cystogenic diseases.
\end{abstract}

Conflict of interest: The author has declared that no conflict of interest exists.

Citation for this article: $J$ Clin Invest. 2010; 120(3):660-663. doi:10.1172/JCI42378.
Based on a flood of recent evidence, primary cilia are now heralded as sensory organelles for detection and transmission of a broad range of cues from the extracellular environment of cells (1), including mechanical and chemical information as diverse as fluid flow in kidneys, mechanical bone deformation, and light and odorant detection (2). By processing such physical and chemical information from the environment into molecular signals in development and postnatal growth and homeostasis, cilia can affect cell differentiation and polarity and cell cycle control (3).

Genetic damage to primary cilia results in a spectrum of perplexing disorders with seemingly disparate manifestations, now classified as ciliopathies. A growing list of bona fide ciliopathies at present includes Bardet-Biedl syndrome (BBS), nephronophthisis (NPHP), and Senior-Loken syndrome (SNLS), just to name a few. Common 


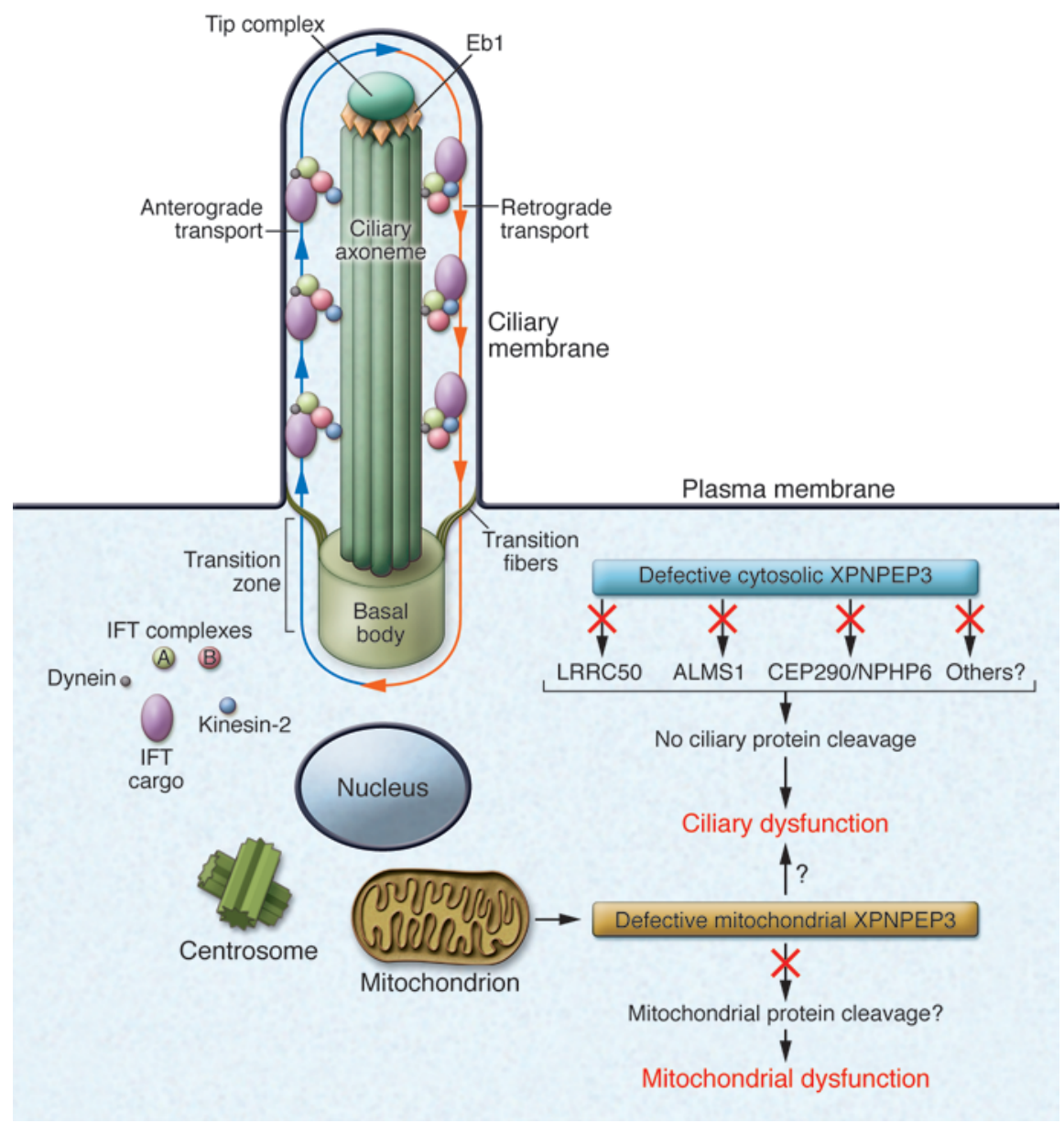

Figure 1

Ciliary and/or mitochondrial dysfunction as consequences of defective ciliary and mitochondrial XPNPEP3. Cilia consist of a microtubule-based axoneme enveloped by a specialized plasma membrane. The basal body is a microtubule-organizing center that anchors the axoneme. The transition zone (composed of transition fibers) filters molecules that enter or exit the cilium at the junction of the basal body. Axonemal and membrane components are transported in raft macromolecular particles (complexes A and B) by intraflagellar transport (IFT) along the axonemal doublet microtubules toward the tip complex, supported by microtubule end binding protein 1 (Eb1). Anterograde IFT toward the cilial tip is driven by heterotrimeric kinesin-2. Retrograde IFT back to the cell body occurs via the cytoplasmic motor protein dynein. As suggested in the study by O'Toole et al. in this issue of the $J C I$ (13), a cytosolic 48-kDa form of XPNPEP3 may mediate enzymatic cleavage of known cystic-disease proteins ALMS1, LRRC50, and CEP290/ NPHP6, and/or other yet unknown ciliary proteins, possibly regulating ciliary targeting or transport. O'Toole et al. report that loss of substrate cleavage, which is associated with defective XPNPEP3, leads to ciliary dysfunction. Similarly, loss of mitochondrial 51-kDa XPNPEP3 peptidase activity may result in failure to cleave yet unknown mitochondrial XPNPEP3 substrates and mitochondrial dysfunction. However, it is unclear at this time whether or how mitochondrial XPNPEP3 defects affect ciliary function. Image adapted with permission from Journal of the American Society of Nephrology (9).

clinical features of ciliopathies include the hallmark renal cysts as well as liver disease, laterality defects, polydactyly, cognitive dysfunction, retinal degeneration, skeletal bone defects, and obesity among others (4).

Cilia are composed of a plasma membrane sheath, enveloping a microtubulebased axoneme that extends from the basal transport processes includes, among others, components of several major signaling pathways, including sonic hedgehog receptors $(6,7)$ and WNT receptors $(8)$. Thus, primary cilia modulate activities of major developmental signaling pathways involved in cell cycle control, differentiation, and planar cell polarity, and various ciliopathy-associated problems can be attributed to disrupted hedgehog and WNT signaling $(2,9,10)$.

\section{The growing spectrum of NPHP pathways}

Among the ciliopathies, NPHP is an autosomal recessive cystic kidney disease characterized by the triad of corticomedullary cysts, tubular basement membrane disruption, and tubulointerstitial nephropathy (9). Ten causative NPHP genes (NPHP1NPHP9 and NPHP11) have been identified by positional cloning and a functional candidate gene approach, and their gene products, known as nephrocystins, localize to primary cilia (nephrocystins 1-5) and to basal bodies and centrosomes (nephrocystins 2-6, 8, and 9) $(9,11)$. An exception is NPHP7 (also known as GLI-similar protein 2 [GLIS2]), which is similar to the GLI family of transcription factors that are downstream targets of hedgehog signaling (12).

In a new and stunning twist on ciliopathies, in their new study in this issue of the JCI, O'Toole et al. report the presence of homozygous frameshift and splice-site mutations in the XPNPEP3 gene (which encodes X-prolyl aminopeptidase 3) in two families with an NPHPlike phenotype (13). Surprisingly, the XPNPEP3 protein has an N-terminal mitochondrial-targeting signal (MTS), and using recombinant expression in inner medullary collecting duct cells (IMCDs) of XPNPEP3-GFP chimeric proteins carrying the MTS, the authors confirmed the exclusive mitochondrial but not ciliary localization of the XPNPEP3 protein. While suppression of xpnpep3 in zebrafish phenocopied the developmental phenotypes observed in zebrafish ciliopathy morphants, the authors were surprised to find that expression of a cytosolic form of human XPNPEP3 lacking the MTS was able to rescue the ciliarelated phenotype, indicating that mitochondrial localization of XPNPEP3 is not essential for its ciliary function.

XPNPEP3 belongs to the aminopeptidase P family of metallo-exopeptidases that specifically remove the $\mathrm{N}$-terminal amino acid 


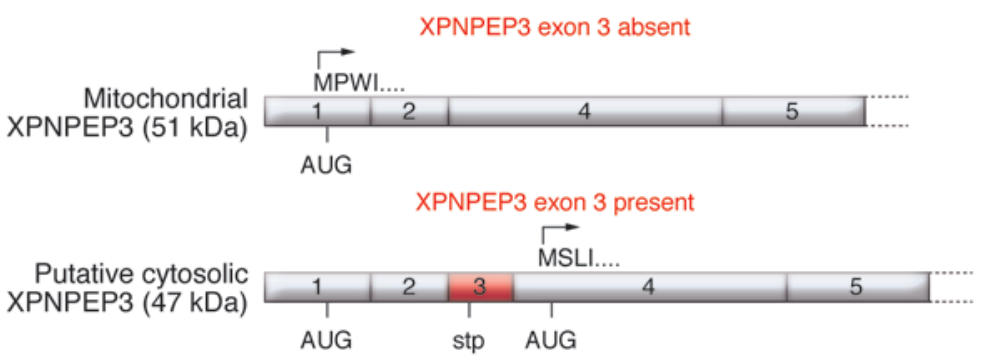

\section{Figure 2}

Two alternative splice forms of XPNPEP3 exist in the kidney. If exon 3 of XPNPEP3 is absent, the XPNPEP3 gene product starts with amino acid sequence MPWI and contains an N-terminal MTS that targets the protein to mitochondria. Exon 3 contains a stop codon (stp) in-frame, resulting in premature termination and removal of the MTS-containing gene product. The alternative XPNPEP3, lacking MTS, is translated from exon 4 AUG, possesses the starting amino acid sequence MSLI, and is likely localized to the cytosol. Mitochondrial XPNPEP3, i.e., the exon 3-spliced form, was used in the localization studies reported in this issue by O'Toole et al. (13).

from peptides that have a proline residue in the second position after methionine (14). XPNPEP2 is a ubiquitous cell-surface membrane-bound peptidase that is thought to degrade peptides filtered by renal epithelium brush border membranes but may also be involved in bradykinin metabolism in endothelial cells (15). In contrast to XPNPEP2, physiological substrates or functions have not yet been identified for XPNPEP1 (which is a soluble cytosolic isoform) or XPNPEP3 (which is known to occur in two alternative splice forms) (Figure 2) (14). In contrast with the mitochondrial form of XPNPEP3, the cytosolic variant lacks the MTS (Figure 2). Importantly, both splice variants are expressed in kidney (14).

\section{Proof of principle for the XPNPEP3 enzyme}

How then may a poorly characterized enzyme with mitochondrial localization in distal tubules and intercalated collecting duct cells in human kidney cause cystic renal disease similar to NPHP, a bona fide ciliopathy? O'Toole et al. examined mitochondrial and respiratory chain functions in muscle biopsies and fibroblasts in affected individuals of the aforementioned kindreds (13). Mitochondriopathy with childhood end-stage renal disease and severe extrarenal manifestations (seizures, cardiomyopathy, and pancreatitis) was observed in two affected individuals in the Turkish kindred, carrying the homozygous frameshift mutation p.N311LfsX5. However, mitochondrial function was normal in affected adults with moderate renal disease from the Finnish kindred, carrying the less severe splice-site mutation p.G453C in XPNPEP3. These find- ings were inconsistent with the mitochondrial dysfunction hypothesis, and together with the observation that a cytosolic form of XPNPEP3 was able to rescue cilia-related phenotypes in the zebrafish studies, these data suggest that the functional defect underlying the NPHP-like renal phenotype might be independent of mitochondria.

The authors offer no explanation for the apparent contradiction between mitochondrial localization and cytosolic function of XPNPEP3 in NPHP-like phenotypes in their study but pursued another lead with acceptable results (13). They examined a pool of 426 likely ciliary protein sequences for candidate XPNPEP3 substrates and identified 51 putative candidates, including three proteins - centrosomal protein 290 kDa/NPHP6 (CEP290/NPHP6), Alstrom syndrome 1 (ALMS1), and leucinerich repeat-containing 50 (LRRC50) - that are known to cause cystic disease (16-18). Indeed, synthetic peptides identical to the $\mathrm{N}$-termini of all three substrate candidates were cleaved as predicted by the recombinant Escherichia coli ortholog of XPNPEP3, ecAPP. A key finding was the demonstration of the authors that an xpnpep3-mediated cleavage-resistant LRRC50 failed, while WT LRRC50 succeeded to rescue the ciliarelated phenotype associated with endogenous $\operatorname{lrc} 50$ suppression in zebrafish. The authors rightfully concluded that loss of cleavage of substrates with ciliary and centrosomal functions may explain some forms of NPHP-like cystic renal disease, but their provocative speculation about an emerging link between mitochondria and ciliary dysfunction deserves further scrutiny. activity of XPNPEP3-mediated enzymatic

\section{Mitochondrial XPNPEP3: a red herring for ciliopathies?}

Is it possible that the mitochondrial localization of XPNPEP3 shown in this report (13) is a red herring when it comes to ciliopathy pathways? The answer may well be affirmative. XPNPEP3 localization studies in the current report were performed exclusively using an XPNPEP3-GFP chimeric construct containing the MTS, which, not surprisingly, localized exclusively to mitochondria when expressed in murine IMCD3 cells. However, two alternative splice variants of XPNPEP3 were previously reported in kidney (14). The alternative splice variant studied by O'Toole et al. lacks exon 3 and initiates translation of a 507-amino acid XPNPEP3 isoform in exon 1 , encoding an N-terminal MTS, which is removed by peptidases after import to mitochondria, resulting in an approximately 51-kDa mature mitochondrial enzyme (Figure 2). In contrast, exon 3 presents a stop codon in-frame to the exon 1 AUG, resulting in premature termination and removal of the truncated protein, and expression of a 428-amino acid isoform of XPNPEP3 devoid of MTS, translated from an AUG site located in exon 4 (14). Indeed, after probing for endogenous XPNPEP3 in IMCD 3 cell lysates, the authors describe, in addition to the $51-\mathrm{kDa}$ mitochondrial form, a second discrete band of XPNPEP3 of approximately $48 \mathrm{kDa}$, which can be predicted from the 428-amino acid cytosolic form of XPNPEP3 (13).

If the $48-\mathrm{kDa}$ band is indeed the predicted cytosolic form of XPNPEP3, then the mitochondrial link may well be a red herring, because the NPHP-like disease observed in some individuals with XPNPEP3 mutations may be attributable to loss of proper cleavage of ciliary proteins by defective cytosolic XPNPEP3, as suggested by the overwhelming evidence from zebrafish studies (13). Of course, the same defect(s) in the mitochondrial form of XPNPEP3 may underlie some of the observed extrarenal manifestations, such as cardiomyopathy and seizures, which may be caused by mitochondrial dysfunction, as demonstrated in one kindred, and independent of ciliary dysfunction.

Perhaps the most intriguing prospect of the exciting study by O'Toole et al. (13) is that the novel discovery of functional mutations in the XPNPEP3 aminopeptidase in individuals presenting with NPHPlike ciliopathy may well lead to the further elucidation of two independent pathways 
associated with a distinct phenotypic spectrum: a cystogenic cilium-dependent pathway, dependent on defective cleavage of ciliary proteins by cytosolic XPNPEP3, and a mitochondriopathy, dependent on defective cleavage of mitochondrial substrates by mitochondrial XPNPEP3. Wow! Future work is needed to define the mitochondrial versus ciliary substrates and roles for hitherto rather obscure, spatially defined metallo-exopeptidases, encoded by a single novel human disease gene, XPNPEP3. Investigators concerned with ciliopathies and mitochondriopathies should stay tuned.

\section{Acknowledgments}

The author is supported by NIH grants 5R01DK056077，5R01DK060043, 5R01DK073960, 3U01DK060995, and 1U01DK085688.

Address correspondence to: Erwin P. Böttinger, Charles R. Bronfman Institute for Personalized Medicine, and Department of Medicine, Mount Sinai School of Medicine, One Gustave L. Levy Pl.,
Box 1003, New York, NY 10029. Phone: 212.241.0800; Fax: 212.849.2643; E-mail: erwin.bottinger@mssm.edu.

1. Pazour GJ, Witman GB. The vertebrate primary cilium is a sensory organelle. Curr Opin Cell Biol. 2003;15(1):105-110.

2. Gerdes JM, Davis EE, Katsanis N. The vertebrate primary cilium in development, homeostasis, and disease. Cell. 2009;137(1):32-45.

3. Singla V, Reiter JF. The primary cilium as the cell's antenna: signaling at a sensory organelle. Science. 2006;313(5787):629-633.

4. Tobin JL, Beales PL. The nonmotile ciliopathies. Genet Med. 2009;11(6):386-402.

5. Scholey JM. Intraflagellar transport motors in cilia: moving along the cell's antenna. J Cell Biol. 2008;180(1):23-29.

6. Rohatgi R, Milenkovic L, Scott MP. Patched1 regulates hedgehog signaling at the primary cilium. Science. 2007;317(5836):372-376.

7. Corbit KC, Aanstad P, Singla V, Norman AR, Stainier DY, Reiter JF. Vertebrate smoothened functions at the primary cilium. Nature. 2005;437(7061):1018-1021.

8. Gerdes JM, Katsanis N. Ciliary function and Wnt signal modulation. Curr Top Dev Biol. 2008;85:175-195.

9. Hildebrandt F, Attanasio M, Otto E. Nephronophthisis: disease mechanisms of a ciliopathy. JAm Soc Nephrol. 2009;20(1):23-35.

10. Lancaster MA, et al. Impaired Wnt-beta-catenin signaling disrupts adult renal homeostasis and leads to cystic kidney ciliopathy. Nat Med.
2009;15(9):1046-1054.

11. Otto EA, et al. Hypomorphic mutations in meckelin (MKS3/TMEM67) cause nephronophthisis with liver fibrosis (NPHP11). J Med Genet. 2009;46(10):663-670

12. Attanasio M, et al. Loss of GLIS2 causes nephronophthisis in humans and mice by increased apoptosis and fibrosis. Nat Genet. 2007; 39(8):1018-1024.

13. O'Toole $\mathrm{J}$, et al. Individuals with mutations in $X P N P E P 3$, which encodes a mitochondrial protein, develop a nephronophthisis-like nephropathy. J Clin Invest. 2010;120(3):791-802.

14. Ersahin C, Szpaderska AM, Orawski AT, Simmons WH. Aminopeptidase P isozyme expression in human tissues and peripheral blood mononuclear cell fractions. Arch Biochem Biophys. 2005;435(2):303-310.

15. Kim KS, Kumar S, Simmons WH, Brown NJ. Inhibition of aminopeptidase $\mathrm{P}$ potentiates wheal response to bradykinin in angiotensin-converting enzyme inhibitor-treated humans. J Pharmacol Exp Ther. 2000;292(1):295-298.

16. Sayer JA, et al. The centrosomal protein nephrocystin- 6 is mutated in Joubert syndrome and activates transcription factor ATF4. Nat Genet. 2006; 38(6):674-681.

17. Li G, et al. A role for Alstrom syndrome protein, alms1, in kidney ciliogenesis and cellular quiescence. PLoS Genet. 2007;3(1):e8.

18. Sullivan-Brown J, et al. Zebrafish mutations affecting cilia motility share similar cystic phenotypes and suggest a mechanism of cyst formation that differs from pkd2 morphants. Dev Biol. 2008;314(2):261-275.

\title{
Resisting arrest: a switch from angiogenesis to vasculogenesis in recurrent malignant gliomas
}

\author{
Jeffrey P. Greenfield,1,2 William S. Cobb,,1,2 and David Lyden ${ }^{1,3}$
}

${ }_{1}^{1}$ Pediatric Brain Tumor Research and the Children's Cancer and Blood Foundation Laboratories, Champalimaud Metastasis Programme,

2Department of Neurosurgery, and ${ }^{3}$ Department of Pediatrics, Weill Cornell Medical College and Memorial Sloan-Kettering Cancer Center, New York, New York.

\begin{abstract}
The cellular and molecular events that initiate and promote malignant glioma development are not completely understood. The treatment modalities designed to promote its demise are all ultimately ineffective, leading to disease progression. In this issue of the JCI, Kioi et al. demonstrate that vasculogenesis and angiogenesis potentially play distinct roles in the etiology of primary and recurrent malignant gliomas, suggesting that patient therapy should perhaps be tailored specifically against the predominant vasculature pathway at a given specific stage of gliomagenesis.
\end{abstract}

Formation of new blood vessels is an essential component of malignant glioma development and progression. As the mechanisms underlying this crucial element of tumor growth are gradually elucidated, it has become increasingly evident that this vascularity arises through multiple mechanisms, depending on the stage of the

Conflict of interest: The authors have declared that no conflict of interest exists.

Citation for this article: J Clin Invest. 2010; 120(3):663-667. doi:10.1172/JCI42345. tumor and possibly the therapies utilized to combat tumor growth. With unregulated cell proliferation and growing tumor size, there is a need for greater oxygen supply to sustain this growth. As mounting cellular metabolism outstrips the oxygen supply made available via the existing vasculature, hypoxia ensues, triggering the process that has been dubbed the "angiogenic switch." While the additional blood supply initially may be obtained simply by co-option of preexisting vessels, the increasing hypoxia eventually necessitates angiogenesis - the sprouting of local vessels via proliferation of existing endothelial cells. Alternatively, vasculogenesis may occur as factors released from tumor cells increase recruitment of circulating endothelial precursor cells or bone marrow-derived hematopoietic cells, also resulting in the formation of new vessels to supply the tumor. Understanding the balance between angiogenesis and vasculogenesis lies at the very core of elucidating how tumors grow, and is crucial to the development of anti-angiogenic therapies. A better comprehension of this process may also allow for the design of new, more effective therapies to target the pathways tumors employ to sustain their growth. In the current issue of the JCI, Kioi et al. (1) suggest that vasculogenesis - but not angiogenesis - is at the center of the revascularization that occurs during glioma recurrence. In clinical terms, their findings suggest that when gliomas recur after irradiation, as they 